Original article

\title{
Selective mediastinal node irradiation in non-small cell lung cancer in the IMRT/VMAT era: How to use E(B)US-NA information in addition to PET-CT for delineation?
}

\author{
Stephanie T. Peeters ${ }^{\mathrm{a}}$, Christophe Dooms ${ }^{\mathrm{b}}$, Angela Van Baardwijk ${ }^{\mathrm{c}}$, Anne-Marie C. Dingemans ${ }^{\mathrm{d}}$, \\ Hanneke Martinussen ${ }^{\mathrm{d}}$, Johan Vansteenkiste ${ }^{\mathrm{b}}$, Herbert Decaluwé ${ }^{\mathrm{e}}$, Paul De Leyn ${ }^{\mathrm{e}}$, Jonas Yserbyt ${ }^{\mathrm{b}}$, \\ Kristiaan Nackaerts ${ }^{\mathrm{b}}$, Walter De Wever ${ }^{\mathrm{f}}$, Christophe M. Deroose ${ }^{\mathrm{g}}$, Dirk De Ruysscher ${ }^{\mathrm{a}, \mathrm{c}, *}$

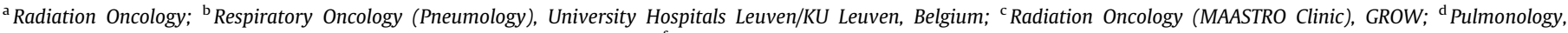 \\ Maastricht University Medical Center, The Netherlands; ${ }^{\mathrm{e}}$ Thoracic Surgery; ${ }^{\mathrm{f}}$ Radiology; and ${ }^{\mathrm{g}}$ Nuclear Medicine, University Hospitals Leuven/KU Leuven, Belgium
}

\section{A R T I C L E I N F O}

\section{Article history:}

Received 17 May 2016

Received in revised form 21 May 2016

Accepted 24 May 2016

Available online $\mathrm{xxxx}$

\section{Keywords:}

Radiotherapy

Non-small cell lung cancer (NSCLC)

Endobronchial ultrasound (EBUS)

Intensity-modulated radiotherapy (IMRT)

Volumetric arc therapy (VMAT)

PET-CT based delineation

\begin{abstract}
A B S T R A C T
Background: FDG-PET-CT-based selective lymph node (LN) irradiation is standard using 3D-conformal techniques for locally advanced NSCLC. With newer techniques (intensity-modulated/volumetric-arc therapy (IMRT/VMAT)), the dose to non-involved adjacent LN decreases, which raises the question whether FDG-PET-CT-delineation is still safe. We therefore evaluated the impact of adding linear endosonography with needle aspiration (E(B)US-NA) to FDG-PET-CT in selective nodal irradiation. Methods: Based on literature data on sensitivity and specificity of E(B)US-NA in FDG-PET-CT-staged NSCLC, false negative (FN) rates for different constellations of CT, PET and E(B)US-NA were calculated. The algorithm was tested on consecutive patients with N2/N3 disease referred for radiotherapy in Leuven and Maastricht.

Results: An algorithm determining when to include LN in the GTV is proposed, based on data from 5 meta-analyses. Adding E(B)US-NA to FDG-PET-CT decreases the FN-rate, but for PET-positive and E(B) US-negative LN, FN rates are still 14-16\%. In Leuven 520 LN were analyzed, in Maastricht 364 LN; with E(B)US-NA a geographical miss was avoided in $2(2 / 40=5 \%)$ and $1(1 / 28=4 \%)$ patients, respectively. Conclusions: E(B)US-NA in addition to FDG-PET-CT for mediastinal staging decreases the risk of a geographical miss with $4-5 \%$. The impact of this small decrease on survival is unknown. The proposed algorithm may guide the radiation oncologist when to include LN in the nodal GTV.
\end{abstract}

(C) 2016 Published by Elsevier Ireland Ltd. Radiotherapy and Oncology xxx (2016) xxx-xxx
Lung cancer is the most common cancer worldwide [1]. The prognosis of patients with locally advanced (LA) or stage III nonsmall cell lung cancer (NSCLC) has improved over the last two decades. The 5 year overall survival is about $30 \%$ [2]. For patients in good general condition, stage III NSCLC is treated with a combined modality approach. The standard treatment for many of these patients is chemoradiation [2]. It has been shown that it is safe to omit irradiation of elective lymph nodes (LN) when using FDG-PET-CT scan to determine the target LN [3,4]. However, this has only been shown for three-dimensional conformal radiation therapy (3D-CRT) techniques. The introduction of intensitymodulated radiotherapy (IMRT) and volumetric arc therapy (VMAT) allowed more shaped distributions and sharper dose gradients than previously possible. In particular for lung cancer

* Corresponding author at: Maastro Clinic, Dr. Tanslaan 12, 6229 ET Maastricht The Netherlands.

E-mail address: dirk.deruysscher@maastro.nl (D. De Ruysscher). patients where the tumor is in close proximity to an organ at risk, or where the target volume includes a large volume of an organ at risk, IMRT or VMAT may potentially be beneficial in minimizing normal tissue toxicity and in adequately covering the target volume $[5,6]$. One of the major concerns however is the decrease in incidental nodal irradiation with IMRT compared with 3D-CRT [7]. With 3D-CRT, adjacent LN not included in the target volume still receive a substantial radiation dose. There are indications that the incidental low doses to regional uninvolved LN contribute to the low regional recurrence rates $[3,8]$. Therefore, the question arises whether selective nodal irradiation based on FDG-PET-CT is still safe when using modern techniques such as IMRT or VMAT.

Linear endosonography (endobronchial ultrasound (EBUS) and/ or esophageal ultrasound (EUS)) with needle aspiration (E(B)US$\mathrm{NA}$ ) is a technique that can give additional pathology-based information on whether LN are malignant or not [9]. With a sensitivity of $>90 \%$ and specificity of $100 \%$ it has been shown to be an accurate 
tool in lung cancer staging in patients with enlarged LN on CT and/ or PET-positive LN $[10,11]$. We therefore evaluated the impact of adding E(B)US-NA-mapping of the mediastinal LN to PET-CT on avoidance of a geographical miss, and on the size of nodal gross tumor volume (GTV) in patients treated with radiotherapy.

The aim of this study was first to make a practical algorithm for daily clinical practice on when to include LN in the GTV in locally advanced NSCLC. This algorithm was based on literature data that included sensitivity and specificity data of E(B)US-NA in FDG-PETCT staged NSCLC. Second, we wanted to test this algorithm on a patient group and analyze how E(B)US-NA mapping impacts on the number of LN considered to be malignant, and on the nodal GTV. First we used a patient group treated at the University Hospitals of Leuven, and second an independent patient group treated at MAASTRO clinic Maastricht.

\section{Methods and materials}

\section{Algorithm}

We first retrieved the expected prevalence for different results of CT (enlarged vs. normal-sized) and PET-scan (positive vs. negative) from literature. We analyzed mediastinal and hilar lymph nodes (LN) together, as the sensitivity of E(B)US-NA to detect malignancy in hilar LN is similar to its performance in mediastinal LN [11]. Secondly, false negative (FN) rates of LN for different constellations of PET, CT and E(B)US-NA based on these literature data were calculated, to evaluate the safety of excluding LN based on CT, PET and E(B)US-NA findings. FN rates were calculated since they have the largest consequence on geographical miss.

Our aim was to propose a practical algorithm that can guide a radiation-oncologist when to include LN in the nodal gross tumor volume (GTV). This algorithm provides the risk of involvement of a LN based on FDG-PET-CT scan and E(B)US-NA.

\section{Patients Leuven}

Consecutive NSCLC-patients referred for radiotherapy (RT) with curative intent in 2012 and 2013 with N2 or N3 disease on FDG-PET-CT scan and who were subjected to E(B)US-NA were included. All stage III NSCLC patients referred for RT have a FDG-PET-CT scan and E(B)US-NA with full mapping of mediastinal LN before RT-planning. Details of the FDG-PET-CT procedures can be found in the Supplementary file. All patient data are entered in a prospective database. This is a retrospective study on patients from this database.

LN stations 1, 2R, 2L, 3 (=3A + 3P), 4R, 4L, 5, 6, 7, 8, 9, 10-11L, 10$11 \mathrm{R}$ (=13 LN stations) were visually analyzed on CT- and PET-scan [13]. On the CT-scan, LN were considered to be positive when the short axis perpendicular to the longest diameter of the LN was $\geqslant 10 \mathrm{~mm}$. On the PET-CT scan, all LN were visually analyzed for FDG-avidity, making use of the written report of the nuclear physician. No SUV-determination was performed because there is no proven added value compared to visual assessment [14].

With E(B)US-NA all LN stations are inspected, and a needle aspiration is done in enlarged or normal sized suspicious LN measuring $>5 \mathrm{~mm}$, when technically feasible [15]. In each patient first the N3 nodes, thereafter the N2 nodes, and finally the N1 nodes are sampled in order to avoid spurious upstaging of the patient through contamination.

\section{Patients Maastricht (external validation)}

An independent dataset was obtained from a prospective database from Maastricht. Inclusion criteria were identical as in Leuven: consecutive NSCLC patients with N2-N3 disease on PET-CT scan, referred for RT, and who were subjected to an E(B)US-NA for mediastinal mapping. LN stations $1-11$ were analyzed on the PET-scan. No information is available on the size of the LN. With E(B)US-NA all LN stations are inspected, and a needle aspiration is done in at least one PET-positive LN to prove N2 or N3 disease. After that, suspicious or enlarged PET-negative LN are sampled, as all PET-positive LN are included in the GTV anyway.

\section{Test of algorithm}

We applied the algorithm on both patient populations. We analyzed the impact of using E(B)US-NA-mapping in addition to FDGPET-CT, first on the number of LN that were considered malignant, and secondly on the number of LN to include in the nodal GTV. Standard delineation of LN nowadays is based on FGD-PET-CT only in 3D-conformal RT and consists of all FDG-PET positive LN [16].

\section{Results}

The expected prevalences of malignant LN (pretest probability) for different constellations of FDG-PET-CT were previously described in a study from Hellwig et al., based on 5 metaanalyses (Fig. 1, 4th column) [17]. When comparing enlarged LN on CT to normal-sized LN, FDG-PET has a higher positive predictive value ( $78 \%$ vs. $70 \%)$, but also a higher false negative (FN) rate $(13 \%$ vs. $6 \%)$.

Using these values, the cancer prevalence was calculated, taking into account the false negative (FN) rate of $\mathrm{E}(\mathrm{B}) \mathrm{US}-\mathrm{NA}$ of $20 \%$ (Fig. 1, 6th column). This FN rate of $20 \%$ was described by Detterbeck et al. and Micames et al. [12,18].

In FDG-PET-negative LN, the addition of E(B)US-NA decreases the FN rate with $10 \%$ for enlarged $\mathrm{LN}$ (from $13 \%$ to $3 \%$, relative reduction of $77 \%$ ), and with $5 \%$ for normal-sized $\mathrm{LN}$ (from $6 \%$ to $1 \%$, relative reduction of $83 \%$ ) (Fig. 1, 4 th and 6th column). An algorithm determining when to include a LN in the GTV is proposed (Fig. 1, last column). Based on this algorithm it is safe to exclude LN that are both PET-negative and E(B)US-negative, regardless of the size of the LN on CT-scan. LN that are PET-negative but $\mathrm{E}(\mathrm{B})$ US-positive should be included in the GTV as the risk of a false positive NA is negligible. For LN that are PET-positive but E(B)USnegative, the risk of having a false negative result is still $14-16 \%$, and they are therefore included in the nodal GTV. This means that compared to PET-based delineation only, the addition of E(B)USNA to PET-CT can increase the volume of the nodal GTV, but not decrease (Fig. 1). Exceptions to this rule are situations where a valid benign reason for PET-positivity is found at pathological examination in case of symmetrical PET-positive LN.

Forty consecutive patients treated at the University Hospitals Leuven were included. We determined the number of patients in which addition of a full E(B)US-NA nodal station mapping before RT resulted in a different nodal GTV using the algorithm. Patient and tumor characteristics are shown in Table 1. Mean age at diagnosis was 64 years. Fifty-five percent of patients had a right-sided primary tumor, $45 \%$ a left-sided. Four patients had a solitary metastasis (two with brain, one with adrenal and one with a renal metastasis) and were considered for treatment with radical intent.

For all patients, the $13 \mathrm{LN}$ stations were visually inspected on CT-scan en PET-scan. This resulted in a total of 520 analyzed LN stations (Fig. 2). $114 \mathrm{LN}$ were enlarged ( $\geqslant 10 \mathrm{~mm}$ ), of which 105 were PET-positive. Twenty normal-sized LN were PET-positive. A total number of $82 \mathrm{LN}$ stations were biopsied with E(B)US-NA, corresponding to a mean of $2.1 \mathrm{LN}$ stations per patient (standard deviation (SD) 0.9; range 1-5). All samples were representative; 49 were malignant, and 30 benign (Fig. 2). In one patient EBUS showed a non-representative sample in $4 \mathrm{~L}$, but with the 


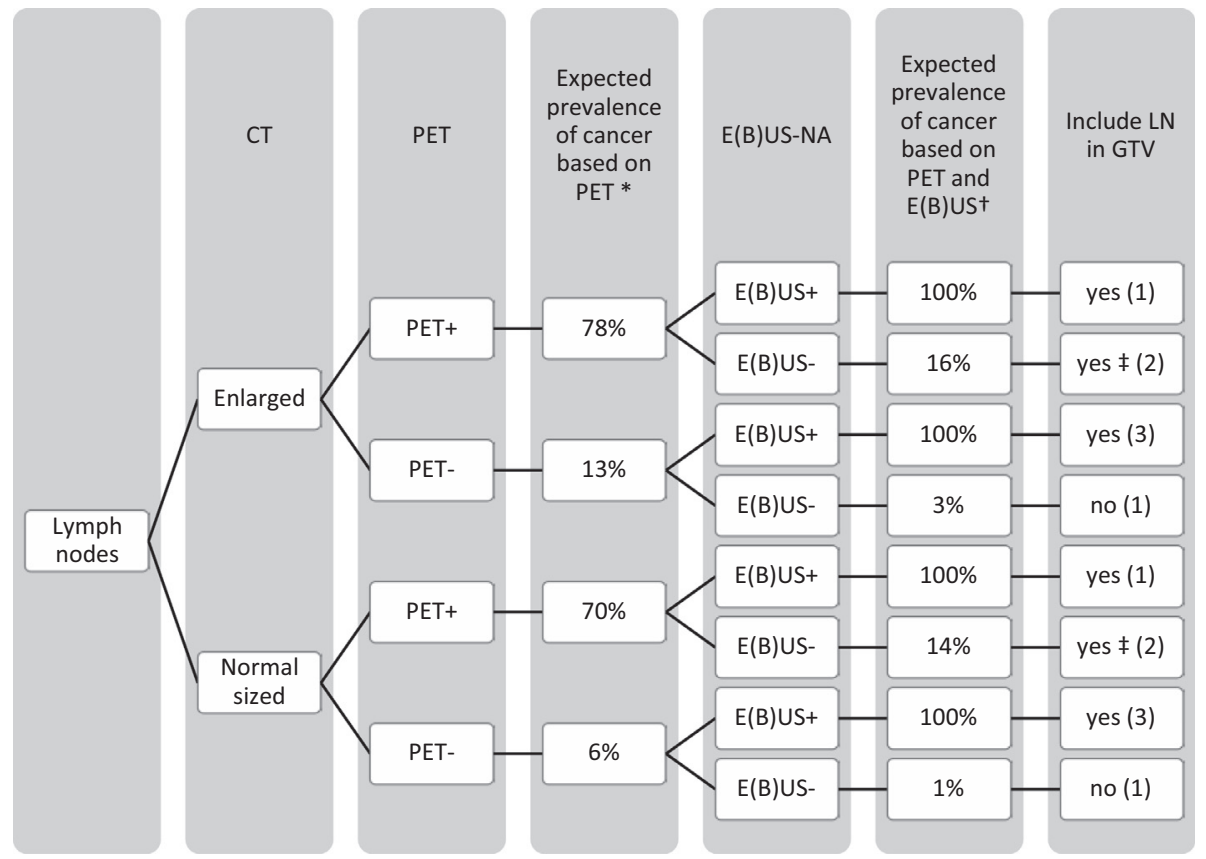

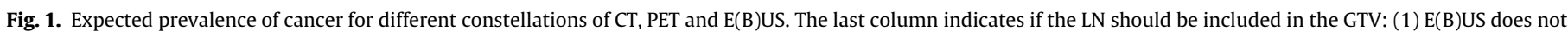

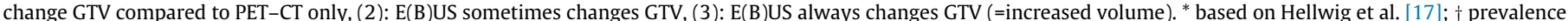

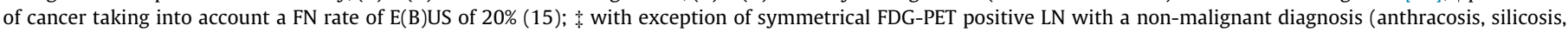

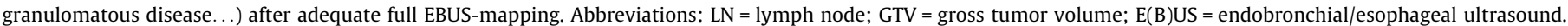

Table 1

Patient and tumor characteristics for Leuven and Maastricht.

\begin{tabular}{|c|c|c|c|}
\hline \multicolumn{2}{|c|}{ Characteristics } & \multirow{2}{*}{ 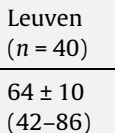 } & \multirow{2}{*}{$\begin{array}{l}\text { Maastricht } \\
(n=28)\end{array}$} \\
\hline Age (years) & $\begin{array}{l}\text { Mean } \pm \text { SD } \\
\text { (Range) }\end{array}$ & & \\
\hline FEV1 (\%) & $\begin{array}{l}\text { Mean } \pm \text { SD } \\
\text { (Range) }\end{array}$ & $\begin{array}{l}79 \pm 21 \\
(40-122) \\
(n=37)\end{array}$ & $\begin{array}{l}80 \pm 22 \\
(32-120) \\
(n=27)\end{array}$ \\
\hline \multirow[t]{2}{*}{ Gender } & Male & 22 & 19 \\
\hline & Female & 18 & 9 \\
\hline Tumor & Right upper lobe & 15 & 11 \\
\hline \multirow[t]{5}{*}{ location } & Right middle lobe & 2 & 1 \\
\hline & Right lower lobe & 5 & 3 \\
\hline & Left upper lobe & 13 & 2 \\
\hline & Left lower lobe & 5 & 9 \\
\hline & Unknown (=T0) & 0 & 2 \\
\hline \multirow[t]{5}{*}{ Histology } & $\begin{array}{l}\text { Squamous cell } \\
\text { carcinoma }\end{array}$ & 10 & 7 \\
\hline & Adenocarcinoma & 24 & 9 \\
\hline & Large cell & 5 & 11 \\
\hline & $\begin{array}{l}\text { Large cell } \\
\text { neuroendocrine }\end{array}$ & 0 & 1 \\
\hline & Unknown & 1 & 0 \\
\hline \multirow[t]{5}{*}{ T-stage } & $\mathrm{T} 1$ & 11 & 3 \\
\hline & $\mathrm{T} 2$ & 13 & 11 \\
\hline & T3 & 9 & 7 \\
\hline & $\mathrm{T} 4$ & 7 & 5 \\
\hline & T0 & 0 & 2 \\
\hline \multirow[t]{2}{*}{$\mathrm{N}^{*}$-stage } & N2 & 26 & 18 \\
\hline & N3 & 14 & 10 \\
\hline \multirow[t]{3}{*}{ M-stage } & M0 & 35 & 28 \\
\hline & M1a & 0 & 0 \\
\hline & M1b & 5 & 0 \\
\hline \multirow[t]{3}{*}{ Stage } & IIIA & 18 & 16 \\
\hline & IIIB & 17 & 12 \\
\hline & IV & 5 & 0 \\
\hline
\end{tabular}

Abbreviations: $\mathrm{SD}=$ standard deviation; FEV1 = Forced Expiratory Volume in $1 \mathrm{~s}$; TNM 7th edition.

$\mathrm{N}$-stage based on FDG-PET-CT

subsequent EUS a representative sample in this $L N$ was obtained. In 2 patients, E(B)US-NA detected a malignant LN not enlarged on CT-scan and not FDG-avid on the PET-scan. Thus, a geographical miss was avoided in 2 patients $(5.0 \%$; $95 \%$ confidence interval $(\mathrm{CI})$ : $0.0 \%-11.8 \%$ ). The first patient was staged as N2 with FDG-PET, but had a N3 LN in contralateral station 4 . The second patient had a normal-sized PET-negative $\mathrm{LN}$ in station 7 malignant with $\mathrm{E}(\mathrm{B})$ US-NA. The time between PET-CT-scan and E(B)US-NA in these 2 patients was 8 and 5 days, respectively. None of the enlarged and PET-negative LN that were biopsied with E(B)US-NA were malignant in this population.

E(B)US-NA was negative in 15 PET-positive LN (11 enlarged and 4 normal-sized) from 9 patients. In 6 of these patients, the LN were described as slight to moderately hypermetabolic whereas the primary tumor was intensely FDG-avid; in one these 6 patients dust laden macrophages were found at pathology. In 3 patients signs of lung inflammation or infection were seen on CT-scan and/or PET-scan.

We determined the incidence of patients in which addition of $\mathrm{E}$ (B)US-NA resulted in a different nodal GTV on an independent external validation cohort of patients treated in Maastricht. Twenty-eight patients with N2 or N3 disease and referred for RT were included in this study. Patients and tumor characteristics are shown in Table 1. Fig. 3 shows the results of using the algorithm, without taking into account the $\mathrm{LN}$ size. A total number of 37 LN were investigated with E(B)US-NA, with a mean of 1.3 LN per patient (SD 0.6; range 1-3). Five $L N$ were not representative at pathological examination. In this patient population, a geographical miss (PET negative and E(B)US-NA positive) was avoided in one patient $(3.6 \%$; $95 \% \mathrm{CI}: 0.0-10.4 \%$ ) by adding $\mathrm{E}(\mathrm{B}) \mathrm{US}-\mathrm{NA}$ to the FDG-PET-CT scan. This patient had a N3 contralateral hilar adenopathy diagnosed with $\mathrm{E}(\mathrm{B}) \mathrm{US}-\mathrm{NA}$.

\section{Discussion}

In this study we propose a practical algorithm that defines when to include LN in the GTV for radiotherapy delineation, based on literature data on sensitivity and specificity of $E(B)$ US-NA in 


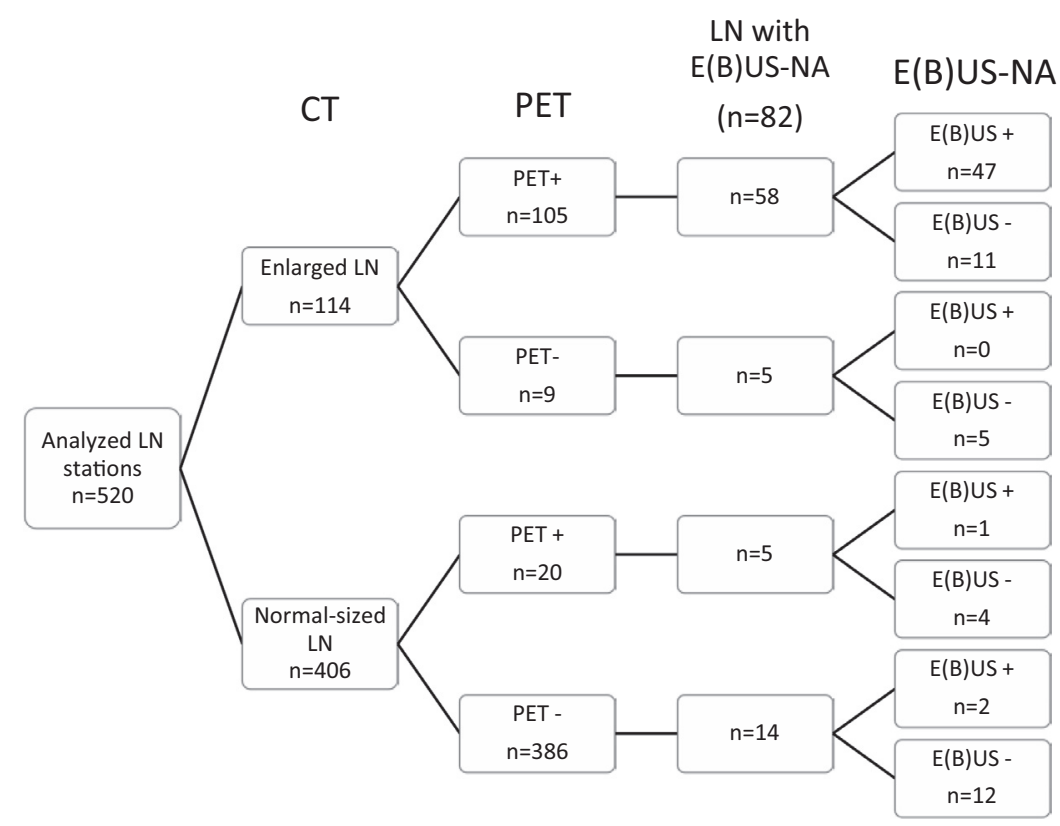

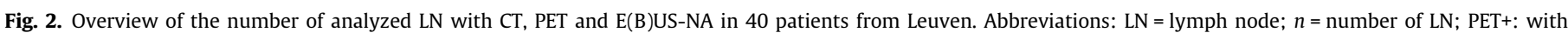

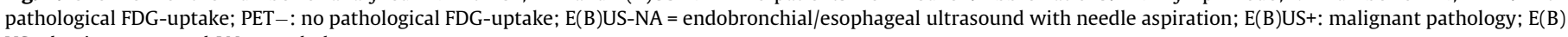
US-: benign or normal LN at pathology.

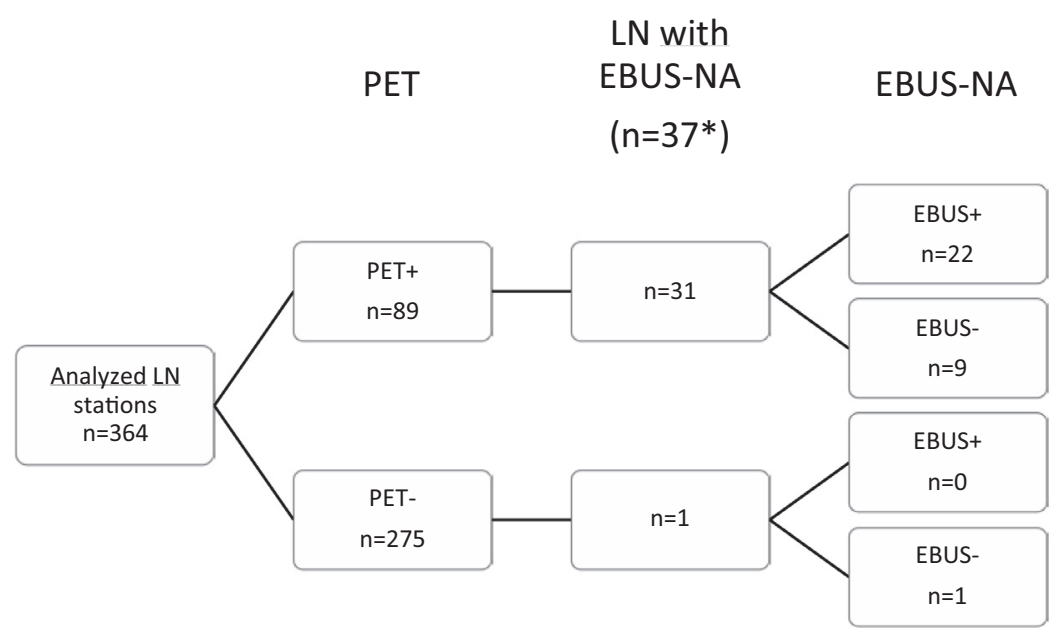

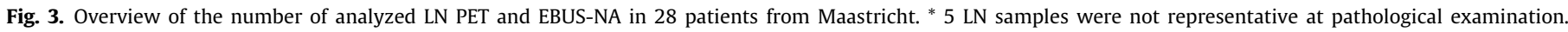
Abbreviations: $\mathrm{LN}=$ lymph node; PET+: with pathological FDG-uptake; PET-: no pathological FDG-uptake; $n=$ number of LN.

FDG-PET-CT staged locally advanced NSCLC (Fig. 1). This overview can also be used as a guide during multidisciplinary discussions to decide whether further diagnostic procedure for nodal evaluation is necessary. We further give some general recommendations on the use of $E(B) U S-N A$ and nodal irradiation that can be used by the radiation oncologist in daily clinical practice (Table 2). This algorithm and these recommendations are of special interest when using newer techniques such as IMRT or VMAT. When treating NSCLC with 3D-CRT, only FDG-PET positive lymph nodes (LN) are included in the gross tumor volume (GTV) [16], as it has previously been shown that PET-based selective nodal irradiation is a safe strategy [3,4]. An important contributive factor for the few observed isolated nodal failures with 3D-CRT is the incidental irradiation of uninvolved mediastinal lymph node areas located in the proximity of the GTV $[7,8]$. But, with newer techniques such as IMRT or VMAT, the dose to non-involved adjacent LN decreases $[3,8]$, which raises the question whether FDG-PET-CT-based delin- eation is still safe [7]. Addition of E(B)US-NA to an integrated FDGPET-CT scan increases the accuracy of mediastinal LN-staging [19]. In clinical practice however, invasive staging is not always routinely done to help defining radiation volumes and all lymph node stations are not readily accessible. We therefore evaluated the impact of adding E(B)US-NA to FDG-PET-CT in the specific context of selective nodal irradiation.

We calculated that addition of E(B)US-NA decreases the false negative rate of a FDG-PET-CT scan, which drops with $10 \%$ for enlarged LN (from $13 \%$ to $3 \%$ ) and with $5 \%$ for normal-sized LN (from 6\% to $1 \%$ ) (Fig. 1). For PET-positive but E(B)US-NA-negative LN however, we calculated that the false negative rate of $\mathrm{E}(\mathrm{B}) \mathrm{US}$ NA is still $14 \%-16 \%$, which we consider to be too high to be omitted from the GTV, except if another valid benign reason for PETpositivity is found at pathological examination. Only in this latter situation the volume of the GTV will be smaller compared with FDG-PET-based delineation only [16]. In all other situations the 
Table 2

Summary of recommendations for nodal GTV delineation based on FDG-PET-CT and E(B)US-NA in daily clinical practice.

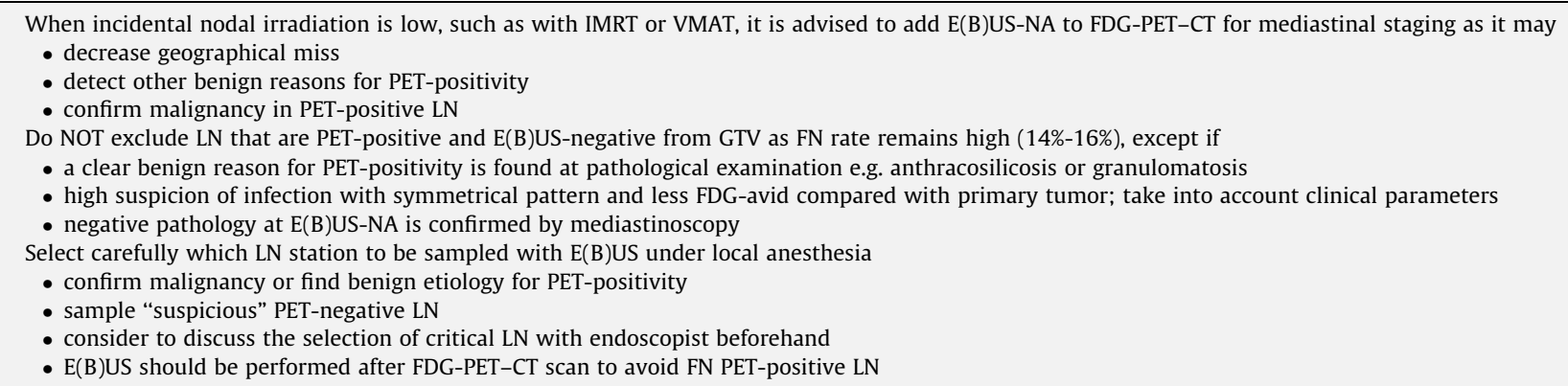

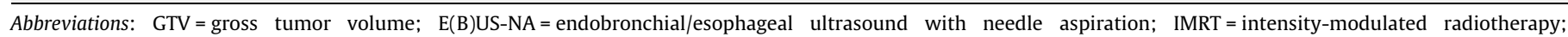
VMAT = volumetric arc therapy; $\mathrm{FN}=$ false negative.

GTV volume will be identical or increased. Pathology-confirmed LN should obviously be included in the nodal GTV. Only LN that are both PET negative and E(B)US-NA-negative can safely be omitted from the GTV, independently from the size of the LN.

This algorithm was first tested on 40 patients from Leuven, where the addition of E(B)US-NA to FDG-PET-CT resulted in avoidance of a geographical miss in 2 patients (5\%; $95 \%$ CI $0-12 \%)$. This result was externally validated in 28 patients from Maastricht where a geographical miss was avoided in 1 patient $(4 \%$; $95 \% \mathrm{CI}$ $0-10 \%)$. However, the question remains whether this decrease of $4-5 \%$ is relevant, given the wide confidence intervals. On the one hand, stage III NSCLC patients have dismal prognosis, and therefore any gain in local control may seem relevant. On the other hand most relapses occur as distant metastases, often in combination with a locoregional recurrence. In those situations nodal failures are not relevant for survival. For these 3 patients with a geographical miss without E(B)US-NA, we calculated the decrease in dose to this missed LN if it would have been excluded from the total PTV in the IMRT plans (results not shown). The mean dose to PTV of this LN decreased from 66 Gy to $41 \mathrm{~Gy}$. Obviously no conclusions can be drawn from these 3 patients concerning the effect on dose to the $\mathrm{LN}$.

Another reason for adding E(B)US-NA to PET-CT is that a benign reason for PET-positivity, such as anthrocosilicosis may be found, allowing to exclude LN from the GTV (Table 2). In the patient group from Leuven, this was the case in 1 patient. In this patient the LN was less FDG-avid compared with the primary tumor and dust-laden macrophages were found. Therefore, caution is needed when using this algorithm as it does not take into account other factors such as the intensity of the nodal PET-positivity compared to the primary tumor or the spatial pattern and/or number of PET-positive LN. The co-existence of inflammatory lung disease (COPD, granulomatous disorders, hypersensitivity pneumonitis, pulmonary fibrosis, cryptogenic organizing pneumonia...) may influence the clinical interpretation of the nodal PET-positivity. For example, the radiation oncologist can decide to omit mediastinal and hilar LN from the GTV in a patient with symmetrical PETpositive $\mathrm{LN}$ in which pathology of the $\mathrm{E}(\mathrm{B}) \mathrm{US}-\mathrm{NA}$ demonstrates a granulomatous reaction in all sampled nodes. Or if inflammation is suspected with normal LN at pathology, prescription of antibiotics followed by a repeat FDG-PET-CT scan may be considered. As clinical interpretation of nodal PET-positivity is sometimes difficult, discussion of these patients at the multidisciplinary board concerning this issue is strongly recommended.

The addition of a videomediastinoscopy when $\mathrm{E}(\mathrm{B}) \mathrm{US}-\mathrm{NA}$ based cytology is negative increases the negative predictive value from $85 \%$ to $93 \%$, but a videomediastinoscopy has a significantly higher morbidity than E(B)US-NA, which is routinely performed under local anesthesia with moderate sedation [19]. In order to avoid additional morbidity we usually do not perform a videomediastinoscopy when E(B)US-NA only shows normal lymphocytes in PET-positive nodes, but include these LN within the GTV in daily clinical practice. This will result in overtreatment in false positive LN, but in most cases will probably not add substantial toxicity from the radiotherapy.

One of the challenges with $\mathrm{E}(\mathrm{B}) \mathrm{US}-\mathrm{NA}$ is the number of sampled lymph nodes. In general, it is recommended to inspect at least 3 mediastinal LN stations (always station 7, ipsilateral station 4 and contralateral station 4), and to sample at least one LN per station, especially suspicious LN and/or FDG-avid lymph nodes [20-22]. In our study the number of E(B)US-NA samples per patient may seem to be relatively low with a mean of 2.1 and 1.3 LN stations sampled per patient in Leuven and Maastricht, respectively. But when an inspected LN station does not contain a visible LN, sampling is impossible. But on the other hand this low number may result in an underestimation of the geographical misses. The lower number in Maastricht compared to Leuven may be due to the policy that only LN that may affect the radiation volume were sampled. According to the proposed algorithm (Fig. 1), FDG-PET-positive LN are included in the GTV anyway, except if E (B)US-NA shows another reason for PET-positivity (e.g. anthracosilicosis). We therefore suggest to sample with E(B)US-NA at least a PET-positive LN to confirm malignancy, and in addition to carefully search for, and sample PET-negative LN stations that may influence nodal stage and GTV.

A possible limitation of the study is that the literature-based expected prevalence of cancer shown in Fig. 1 was only partly observed in the results shown in Fig. 2. Especially for enlarged PET-negative LN a discrepancy was seen. Results of Fig. 1 are based on data from large studies where LN positivity was usually confirmed by mediastinoscopy or thoracotomy, whereas in Fig. 2 data are retrospective (although based on prospectively gathered databases), but reflecting routine clinical situations where the number of pathology samples per patient is lower. We therefore believe that the algorithm can be used for decision-making in daily clinical practice.

Another limitation is that no data on locoregional recurrence and overall survival were presented as the number of patients is too small. The patient data set contained 40 patients in Leuven, and 28 patients in the external dataset from Maastricht. In these patients, a large number of LN were inspected: 13 LN stations on FDG-PET-CT scan (=884 LN stations) and 7 LN stations with E(B) US-NA (=476 LN stations). A total of 119 LN were sampled with E (B)US-NA. We therefore consider the patient data sets large enough to support the proposed concept, but not to analyze locoregional recurrences or overall survival. 
In conclusion, the addition of E(B)US-NA to FDG-PET-CT for mediastinal staging in locally advanced NSCLC decreases the risk of a geographical miss with 4-5\%. Whether addition of $\mathrm{E}(\mathrm{B}) \mathrm{US}-$ NA will impact on survival is however unknown as isolated nodal failures are rare. On the other hand, in daily clinical practice it is sometimes difficult to decide whether a LN should be included in the GTV in case of conflicting results on PET and E(B)US-NA. The algorithm and recommendations are therefore proposed to guide the radiation-oncologist in the delineation of the nodal GTV (Fig. 1, Table 2). LN that are both PET-positive and E(B)US-NAnegative should be included in the GTV as FN-rates remain high, except if a valid benign reason for PET-positivity is found at pathological examination.

\section{Conflict of interest statement}

None of the authors have a conflict of interest to report.

\section{Appendix A. Supplementary data}

Supplementary data associated with this article can be found, in the online version, at http://dx.doi.org/10.1016/j.radonc.2016.05. 023.

\section{References}

[1] Siegel RL, Miller KD, Jemal A. Cancer statistics, 2016. CA Cancer J Clin 2016.

[2] Eberhardt WE, De Ruysscher D, Weder W, et al. 2nd ESMO Consensus Conference in Lung Cancer: locally advanced stage III non-small-cell lung cancer.

[3] De Ruysscher D, Wanders S, van Haren E, et al. Selective mediastinal node irradiation based on FDG-PET scan data in patients with non-small-cell lung cancer: a prospective clinical study. Int J Radiat Oncol Biol Phys 2005:62:988-94.

[4] Belderbos J, Heemsbergen WD, De Jaeger K, et al. Final results of a phase I/II dose escalation trial in non-small-cell lung cancer using three-dimensional conformal radiotherapy. Int J Radiat Oncol Biol Phys 2006;66:126-34.

[5] Bezjak A, Rumble RB, Rodrigues G, et al. Intensity-modulated radiotherapy in the treatment of lung cancer. Clin Oncol (R Coll Radiol) 2012;24:508-20.

[6] Lievens Y, Nulens A, Gaber MA, et al. Intensity-modulated radiotherapy for locally advanced non-small-cell lung cancer: a dose-escalation planning study. Int J Radiat Oncol Biol Phys 2011;80:306-13.

[7] Kepka L, Maciejewski B, Withers RH. Does incidental irradiation with doses below 50 Gy effectively reduce isolated nodal failures in non-small-cell lung cancer: dose-response relationship. Int J Radiat Oncol Biol Phys 2009;73:1391-6.

[8] Kimura T, Togami T, Nishiyama Y, et al. Impact of incidental irradiation on clinically uninvolved nodal regions in patients with advanced non-small-cell lung cancer treated with involved-field radiation therapy: does incidental irradiation contribute to the low incidence of elective nodal failure? Int J Radiat Oncol Biol Phys 2010;77:337-43.

[9] Dooms C, Seijo L, Gasparini S, et al. Diagnostic bronchoscopy: state of the art. Eur Respir Rev 2010;19:229-36.

[10] Gu P, Zhao Y, Jiang L, et al. Endobronchial ultrasound-guided transbronchial needle aspiration for staging of lung cancer: a systematic review and metaanalysis. Eur J Cancer 2009;45:1389-96.

[11] Ernst A, Eberhardt R, Krasnik M, et al. Efficacy of endobronchial ultrasoundguided transbronchial needle aspiration of hilar lymph nodes for diagnosing and staging cancer. J Thorac Oncol 2009;4:947-50.

[12] Micames CG, Mc Crory DC, Pavey DA, et al. Endoscopic ultrasound-guided fineneedle aspiration for non-small cell lung cancer staging: a systematic review and metaanalysis. Chest 2007;131:539-48.

[13] Rusch VW, Asamura H, Watanabe H. The IASLC lung cancer staging project: a proposal for a new international lymph node map in the forthcoming seventh edition of the TNM classification for lung cancer. J Thorac Oncol 2009;4:679-83.

[14] Vansteenkiste JF, Stroobants SG, De Leyn PR, et al. Lymph node staging in nonsmall cell lung cancer with FDG-PET scan: a prospective study on 690 lymph node stations from 68 patients. J Clin Oncol 1998;16:2142-9.

[15] Kang HJ, Hwangbo B, Lee GK, et al. EBUS-centred versus EUS-centred mediastinal staging in lung cancer: a randomised controlled trial. Thorax 2014;69:261-8.

[16] De Ruysscher D, Faivre-Finn C, Nestle U, et al. European Organisation for Research and Treatment of Cancer recommendations for planning and delivery of high-dose, high-precision radiotherapy for lung cancer. J Clin Oncol 2010;28:5301-10.

[17] Hellwig D, Baum RP, Kirsch C. FDG-PET, PET/CT and conventional nuclear medicine procedures in the evaluation of lung cancer: a systematic review. Nuklearmedizin 2009;48:59-69.

[18] Detterbeck FC, Jantz MA, Wallace M. Invasive mediastinal staging of lung cancer: ACCP evidence-based clinical practice guidelines (2nd edition). Chest 2007; 132:202S-20S.

[19] Annema J, van Meerbeeck J, Rintoul R, et al. Mediastinoscopy versus endosonography for mediastinal nodal staging of lung cancer: a randomized trial. JAMA 2010;304:2245-52.

[20] Dietel M, Bubendorf L, Dingemans AM, Dooms C, Elmberger G, García RC, et al Diagnostic procedures for non-small-cell lung cancer (NSCLC): recommendations of the European Expert Group. Thorax 2016;71:177-84.

[21] De Leyn P, Dooms C, Kuzdzal J, et al. Revised ESTS guidelines for preoperative mediastinal lymph node staging for non-small-cell lung cancer. Eur J Cardiothorac Surg 2014;45:787-98.

[22] Vilmann P, Clementsen PF, Colella S, et al. Combined endobronchial and esophageal endosonography for the diagnosis and staging of lung cancer: European Society of Gastrointestinal Endoscopy (ESGE) Guideline, in cooperation with the European Respiratory Society (ERS) and the European Society of Thoracic Surgeons (ESTS). Endoscopy 2015;47:545-59. 